\title{
Graphene: a promising two-dimensional support for heterogeneous catalysts
}

\author{
Xiaobin Fan* \\ State Key Laboratory of Chemical Engineering, School of Chemical Engineering and Technology, Tianjin University, Tianjin, China
}

Edited by:

Alan Brian Dalton, University of

Surrey, UK

Reviewed by:

Ji-Jun Zou, Tianjin University, China

Kwun Nam Hui, Pusan National

University, South Korea

*Correspondence:

Xiaobin Fan, State Key Laboratory of

Chemical Engineering, School of

Chemical Engineering and

Technology, Tianjin University, 92

Weijin Road, Tianjin 300072, China

e-mail:xiaobinfan@tju.edu.cn

\begin{abstract}
Graphene has many advantages that make it an attractive two-dimensional (2D) support for heterogeneous catalysts. It not only allows the high loading of targeted catalytic species but also facilitates the mass transfer during the reaction processes. These advantages, along with its unique physical and chemical properties, endow graphene great potential as catalyst support in heterogeneous catalysis.
\end{abstract}

Keywords: graphene, support, heterogeneous catalysts, mass transfer, electron transfer
A large variety of carbon materials (activated carbons and carbon blacks, in particular) have been exploited as catalyst supports in heterogeneous catalysis. The advantages of carbon supports can be attributed to their large surface area, inert property that endows their resistance to both acidic and basic media, as well as the convenience to recover the active phase (especially for the supported noble metal catalysts) by direct burning away the carbon supports.

Compared with the traditional carbon materials, as well as other supports such as zeolite, porous silica, and MCM41, graphene possesses many unique properties that make it an attractive support in heterogeneous catalysis. In particular, with a huge surface area (theoretical value of $2630 \mathrm{~m}^{2} / \mathrm{g}$ ) (Stoller et al., 2008), two-dimensional (2D) structures (Rao et al., 2009), a high electron mobility (Castro Neto et al., 2009), and the availability of surface functionalization (Dreyer et al., 2010; Georgakilas et al., 2012), it not only allows the high loading of targeted catalytic species but also achieves exceptional performance in various catalytic applications (Scheuermann et al., 2009). The unique 2D structures of graphene, which can facilitate the mass transfer during the reaction processes should firstly account for the excellent results (Figure 1). To be specific, for the heterogeneous catalysts with common porous supports, there are seven steps in a catalytic reaction process: (1) diffusion of the reactants from the liquid or gas phase to the external surface of the catalyst (external diffusion); (2) diffusion of the reactant from the pore mouth through the catalyst pores to the immediate vicinity of the internal catalytic surface (internal diffusion); (3) adsorption of reactants on the inner catalytic surface; (4) reaction at active sites on the catalyst surface; (5) desorption of the products from the inner surface; (6) diffusion of the products from the interior of the catalyst to the pore mouth at the external surface (internal diffusion); (7) diffusion of the products from the external pellet surface to the bulk fluid (external diffusion). The activity of heterogeneous catalysts is proportional to the active surface area per unit volume of catalyst, provided that transport limitations are not present. However, diffusion control is very common in heterogeneous catalytic processes. So the reaction rate is principally relied on the mass transfer or diffusion steps. As external diffusion can be promoted by raising the stirring intensity and reaction temperature, numerous reactions are restricted to the internal diffusion step, especially when the pore sizes are quite small or just comparable with the reactant molecules. For graphene supported catalyst with good suspending ability in the fluid phase; however, the mass transfer of the reactants to the active sites is unlimited, as the reactants can easily access the catalytic active sites on both sides of the suspended 2D graphene sheets without an internal diffusion process (Ji et al., 2011). Besides the facile mass transfer, the versatile electronic behaviors of graphene also render it great potential for chemical and energy conversion applications, especially in electrocatalytic and photocatalytic processes. As the catalytic species (metal nanoparticles, in particular) are directly distributed on the graphene surface, there should be a strong metal-support interaction. The conductive graphene may cause electronic perturbations as well as constraining the geometries of the dispersed metal catalysts, which may display synergetic promotion effect to catalytic conversion. What is more, the high electron mobility also facilitates the rapid diffusion of electrons. For instance, in electrocatalytic processes, the conversion can be greatly promoted by the rapid electron transfer between the reactant and the electrode with the graphene support. For photocatalytic reactions, graphene serves as a charge carrier transport "highway," mitigating the recombination of charge carriers and leading to an enhanced photocatalytic performance. Consequently, graphene derivatives, such as nitrogendoped graphene that can increase the electrical conductivity of graphene and improve the graphene-metal binding interactions, have been widely exploited in related applications.

These advantages, along with its unparalleled high tensile strength (Dikin et al., 2007), excellent thermal property 


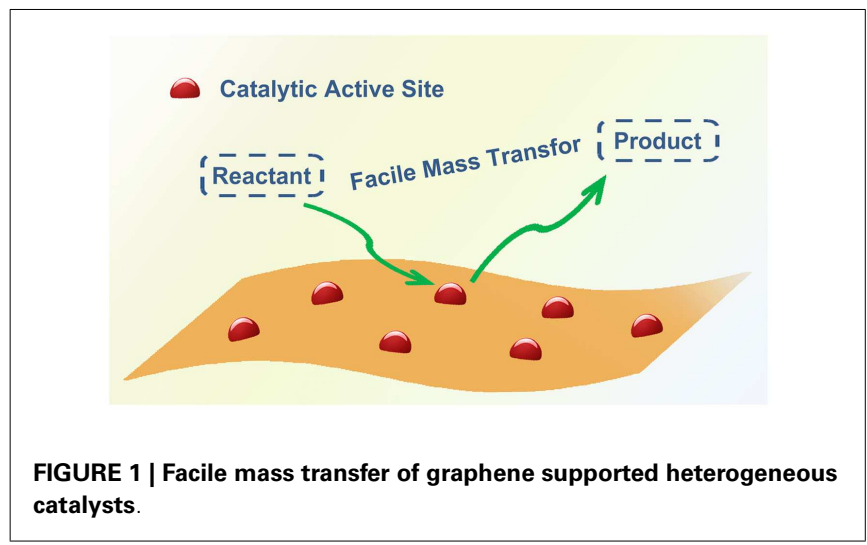

(Balandin et al., 2008), and a high optical transparency (Bae et al., 2010), endows graphene great potential as a catalyst support for the chemical industry, electrochemistry, photocatalysis, energy conversion, energy storage, etc.

\section{REFERENCES}

Bae, S., Kim, H., Lee, Y., Xu, X. F., Park, J. S., Zheng, Y., et al. (2010). Roll-to-roll production of 30-inch graphene films for transparent electrodes. Nat. Nanotechnol. 5, 574-578. doi:10.1038/NNANO.2010.132

Balandin, A. A., Ghosh, S., Bao, W. Z., Calizo, I., Teweldebrhan, D., Miao, F., et al. (2008). Superior thermal conductivity of single-layer graphene. Nano Lett. 8, 902-907. doi:10.1021/nl0731872

Castro Neto, A. H., Guinea, F., Peres, N. M. R., Novoselov, K. S., and Geim, A. K. (2009). The electronic properties of graphene. Rev. Mod. Phys. 81, 109-162. doi:10.1103/RevModPhys.81.109

Dikin, D. A., Stankovich, S., Zimney, E. J., Piner, R. D., Dommett, G. H. B., Evmenenko, G., et al. (2007). Preparation and characterization of graphene oxide paper. Nature 448, 457-460. doi:10.1038/nature06016
Dreyer, D. R., Park, S., Bielawski, C. W., and Ruoff, R. S. (2010). The chemistry of graphene oxide. Chem. Soc. Rev. 39, 228-240. doi:10.1039/b917103g

Georgakilas, V., Otyepka, M., Bourlinos, A. B., Chandra, V., Kim, N., Kemp, K. C., et al. (2012). Functionalization of graphene: covalent and non-covalent approaches, derivatives and applications. Chem. Rev. 112, 6156-6214. doi:10. $1021 / \mathrm{cr} 3000412$

Ji, J. Y., Zhang, G. H., Chen, H. Y., Wang, S. L., Zhang, G. L., Zhang, F. B., et al. (2011). Sulfonated graphene as water-tolerant solid acid catalyst. Chem. Sci. 2, 484-487. doi:10.1039/C0SC00484G

Rao, C. N. R., Sood, A. K., Subrahmanyam, K. S., and Govindaraj, A. (2009). Graphene: the new two-dimensional nanomaterial. Angew. Chem. Int. Ed. 48, 7752-7777. doi:10.1002/anie.200901678

Scheuermann, G. M., Rumi, L., Steurer, P., Bannwarth, W., and Mülhaupt, R. (2009). Palladium nanoparticles on graphite oxide and its functionalized graphene derivatives as highly active catalysts for the Suzuki-Miyaura coupling reaction. J. Am. Chem. Soc. 131, 8262-8270. doi:10.1021/ja901105a

Stoller, M. D., Park, S. J., Zhu, Y. W., An, J. H., and Ruoff, R. S. (2008). Graphene-based ultracapacitors. Nano Lett. 8, 3498-3502. doi:10.1021/nl802558y

Conflict of Interest Statement: The Review Editor Ji-Jun Zou declares that, despite being affiliated to the same institution as author Xiaobin Fan, the review process was handled objectively and no conflict of interest exists. The author declares that the research was conducted in the absence of any commercial or financial relationships that could be construed as a potential conflict of interest.

Received: 15 August 2014; accepted: 31 December 2014; published online: 21 January 2015.

Citation: Fan X (2015) Graphene: a promising two-dimensional support for heterogeneous catalysts. Front. Mater. 1:39. doi: 10.3389/fmats.2014.00039

This article was submitted to Carbon-Based Materials, a section of the journal Frontiers in Materials.

Copyright $\odot 2015$ Fan. This is an open-access article distributed under the terms of the Creative Commons Attribution License (CC BY). The use, distribution or reproduction in other forums is permitted, provided the original author(s) or licensor are credited and that the original publication in this journal is cited, in accordance with accepted academic practice. No use, distribution or reproduction is permitted which does not comply with these terms. 